\title{
Form factors of boundary exponential operators in the sinh-Gordon model
}

\author{
G. Takács* \\ Theoretical Physics Research Group of the Hungarian Academy of Sciences \\ H-1117 Budapest, Pázmány Péter sétány 1/A
}

7th January, 2008

\begin{abstract}
Using the recently introduced boundary form factor bootstrap equations, the form factors of boundary exponential operators in the sinh-Gordon model are constructed. The ultraviolet scaling dimension and the normalization of these operators are checked against previously known results. The construction presented in this paper can be applied to determine form factors of relevant primary boundary operators in general integrable boundary quantum field theories.
\end{abstract}

\section{Introduction}

The investigation of integrable boundary quantum field theories started with the seminal work of Ghoshal and Zamolodchikov [1], who set up the boundary R-matrix bootstrap, which makes possible the determination of the reflection matrices and provides complete description of the theory on the mass shell.

For the calculation of correlation functions, matrix elements of local operators between asymptotic states have to be computed. In a boundary quantum field theory there are two types of operators, the bulk and the boundary operators, where their names indicate their localization point. The boundary bootstrap program, namely the boundary form factor program for calculating the matrix elements of local boundary operators between asymptotic states was initiated in [2]. The validity of form factor solutions was checked in the case of the boundary scaling Lee-Yang model calculating the two-point function using

\footnotetext{
*E-mail: takacs@elte.hu
} 
a spectral sum and comparing it to the prediction of conformal perturbation theory. In [3] the spectrum of independent form factor solutions was compared to the boundary operator content of the ultraviolet boundary conformal field theory and a complete agreement was found. Further solutions of the boundary form factor axioms were constructed and their structure was analyzed for the sinh-Gordon theory at the self-dual point in [4, and for the $A_{2}$ affine Toda field theory in [5]. In the recent paper [6] the validity of the form factor solution conjectured for the unique nontrivial boundary primary field in scaling LeeYang model was tested against truncated conformal space and a spectacular agreement was found.

It is clear from the discussion in [3] that the most interesting open problem of the boundary form factor bootstrap is the identification of the operator corresponding to a given solution. For example, in sinh-Gordon theory there are infinitely many form factor solutions with minimal growth at large rapidities, which can be attributed to the presence of exponential boundary fields. The task undertaken in this paper is to map the space of such minimal solutions, and make their correspondence with exponential fields more precise. It is shown that specific solutions can be selected inside this infinite family, such that their scaling dimension agrees with the prediction of conformal field theory, while their normalization matches the available results on the vacuum expectation value of exponential operators. The construction of these solutions can be generalized to determine form factors of relevant boundary primary fields in any model where the form factors of relevant operators in the bulk theory are known.

The outline of the paper is the following. In section 2 the necessary information about boundary sinh-Gordon theory is presented. Section 3 describes the construction of the form factors solutions which are conjectured to correspond to boundary exponential fields. In section 4 their ultraviolet dimension and normalization is evaluated as a series expansion in the bulk parameter of sinh-Gordon theory, and is shown to be consistent with known results. Section 5 is reserved for the conclusions.

\section{Boundary sinh-Gordon theory}

The sinh-Gordon theory in the bulk is defined by the Lagrangian density

$$
\mathcal{L}=\frac{1}{2}\left(\partial_{\mu} \Phi\right)^{2}-\frac{m^{2}}{b^{2}}(\cosh b \Phi-1)
$$

It can be considered as the analytic continuation of the sine-Gordon model for imaginary coupling $\beta=i b$. The S-matrix of the model is

$$
S(\theta)=-\left(1+\frac{B}{2}\right)_{\theta}\left(-\frac{B}{2}\right)_{\theta}=\left[-\frac{B}{2}\right]_{\theta} \quad ; \quad B=\frac{2 b^{2}}{8 \pi+b^{2}}
$$

where

$$
(x)_{\theta}=\frac{\sinh \frac{1}{2}(\theta+i \pi x)}{\sinh \frac{1}{2}(\theta-i \pi x)} \quad, \quad[x]_{\theta}=-(x)_{\theta}(1-x)_{\theta}=\frac{\sinh \theta+i \sin \pi x}{\sinh \theta-i \sin \pi x}
$$


The minimal bulk two-particle form factor belonging to this S-matrix is [7]

$$
f(\theta)=\mathcal{N} \exp \left[8 \int_{0}^{\infty} \frac{d x}{x} \sin ^{2}\left(\frac{x(i \pi-\theta)}{2 \pi}\right) \frac{\sinh \frac{x B}{4} \sinh \left(1-\frac{B}{2}\right) \frac{x}{2} \sinh \frac{x}{2}}{\sinh ^{2} x}\right]
$$

where

$$
\mathcal{N}=\exp \left[-4 \int_{0}^{\infty} \frac{d x}{x} \frac{\sinh \frac{x B}{4} \sinh \left(1-\frac{B}{2}\right) \frac{x}{2} \sinh \frac{x}{2}}{\sinh ^{2} x}\right]
$$

It satisfies $f(\theta, B) \rightarrow 1$ as $\theta \rightarrow \infty$, and approaches its asymptotic value exponentially fast.

Sinh-Gordon theory can be restricted to the negative half-line with the following action

$$
\begin{aligned}
\mathcal{A}= & \int_{-\infty}^{\infty} d t \int_{-\infty}^{0} d x\left[\frac{1}{2}\left(\partial_{\mu} \Phi\right)^{2}-\frac{m^{2}}{b^{2}}(\cosh b \Phi-1)\right] \\
& +\int_{-\infty}^{\infty} d t M_{0}\left(\cosh \left(\frac{b}{2}\left(\Phi(0, t)-\Phi_{0}\right)\right)-1\right)
\end{aligned}
$$

which maintains integrability [1]. The corresponding reflection factor depends on two continuous parameters and can be written as 8

$$
R(\theta)=\left(\frac{1}{2}\right)_{\theta}\left(\frac{1}{2}+\frac{B}{4}\right)_{\theta}\left(1-\frac{B}{4}\right)_{\theta}\left[\frac{E-1}{2}\right]_{\theta}\left[\frac{F-1}{2}\right]_{\theta}
$$

It can be obtained as the analytic continuation of the first breather reflection factor in boundary sine-Gordon model which was calculated by Ghoshal in [9]. The relation of the bootstrap parameters $E$ and $F$ to the parameters of the Lagrangian is known both from a semi-classical calculation [8, 10] and also in an exact form in the perturbed boundary conformal field theory framework [11].

\section{Boundary form factors in sinh-Gordon theory}

\subsection{The boundary form factor axioms}

The axioms satisfied by the form factors of a local boundary operator were derived in [2] and are listed here without much further explanation. Let us assume that the spectrum contains a single scalar particle of mass $m$, which has a two-particle $S$ matrix $S(\theta)$ (using the standard rapidity parametrization) and a one-particle reflection factor $R(\theta)$ off the boundary, satisfying the boundary reflection factor bootstrap conditions of Ghoshal and Zamolodchikov [1]. For a local operator $\mathcal{O}(t)$ localized at the boundary (located at $x=0$, and parametrized by the time coordinate $t$ ) the form factors are defined as

$$
\begin{aligned}
\text { out }\left\langle\theta_{1}^{\prime}, \theta_{2}^{\prime}, \ldots, \theta_{m}^{\prime}|\mathcal{O}(t)| \theta_{1}, \theta_{2}, \ldots,\right. & \left.\theta_{n}\right\rangle_{\text {in }} \\
& F_{m n}^{\mathcal{O}}\left(\theta_{1}^{\prime}, \theta_{2}^{\prime}, \ldots, \theta_{m}^{\prime} ; \theta_{1}, \theta_{2}, \ldots, \theta_{n}\right) e^{-i m t\left(\sum \cosh \theta_{i}-\sum \cosh \theta_{j}^{\prime}\right)}
\end{aligned}
$$


for $\theta_{1}>\theta_{2}>\ldots>\theta_{n}>0$ and $\theta_{1}^{\prime}<\theta_{2}^{\prime}<\ldots<\theta_{m}^{\prime}<0$, using the asymptotic in/out state formalism introduced in [12]. They can be extended analytically to other values of rapidities. With the help of the crossing relations derived in [2] all form factors can be expressed in terms of the elementary form factors

$$
{ }_{\text {out }}\left\langle 0|\mathcal{O}(0)| \theta_{1}, \theta_{2}, \ldots, \theta_{n}\right\rangle_{\text {in }}=F_{n}^{\mathcal{O}}\left(\theta_{1}, \theta_{2}, \ldots, \theta_{n}\right)
$$

which can be shown to satisfy the following axioms:

I. Permutation:

$$
F_{n}^{\mathcal{O}}\left(\theta_{1}, \ldots, \theta_{i}, \theta_{i+1}, \ldots, \theta_{n}\right)=S\left(\theta_{i}-\theta_{i+1}\right) F_{n}^{\mathcal{O}}\left(\theta_{1}, \ldots, \theta_{i+1}, \theta_{i}, \ldots, \theta_{n}\right)
$$

II. Reflection:

$$
F_{n}^{\mathcal{O}}\left(\theta_{1}, \ldots, \theta_{n-1}, \theta_{n}\right)=R\left(\theta_{n}\right) F_{n}^{\mathcal{O}}\left(\theta_{1}, \ldots, \theta_{n-1},-\theta_{n}\right)
$$

III. Crossing reflection:

$$
F_{n}^{\mathcal{O}}\left(\theta_{1}, \theta_{2}, \ldots, \theta_{n}\right)=R\left(i \pi-\theta_{1}\right) F_{n}^{\mathcal{O}}\left(2 i \pi-\theta_{1}, \theta_{2}, \ldots, \theta_{n}\right)
$$

IV. Kinematical singularity

$$
-i \operatorname{Res}_{\theta=\theta^{\prime}} F_{n+2}^{\mathcal{O}}\left(\theta+i \pi, \theta^{\prime}, \theta_{1}, \ldots, \theta_{n}\right)=\left(1-\prod_{i=1}^{n} S\left(\theta-\theta_{i}\right) S\left(\theta+\theta_{i}\right)\right) F_{n}^{\mathcal{O}}\left(\theta_{1}, \ldots, \theta_{n}\right)
$$

V. Boundary kinematical singularity

$$
-i \operatorname{Res}_{\theta=0} F_{n+1}^{\mathcal{O}}\left(\theta+\frac{i \pi}{2}, \theta_{1}, \ldots, \theta_{n}\right)=\frac{g}{2}\left(1-\prod_{i=1}^{n} S\left(\frac{i \pi}{2}-\theta_{i}\right)\right) F_{n}^{\mathcal{O}}\left(\theta_{1}, \ldots, \theta_{n}\right)
$$

where $g$ is the one-particle coupling to the boundary

$$
R(\theta) \sim \frac{i g^{2}}{2 \theta-i \pi} \quad, \quad \theta \sim i \frac{\pi}{2}
$$

There are also axioms for singularities corresponding to bound states (bulk and boundary), but they are not needed in the sequel.

The general solution of the above axioms can be written as [2]

$$
F_{n}\left(\theta_{1}, \theta_{2}, \ldots, \theta_{n}\right)=H_{n} \frac{Q_{n}\left(y_{1}, y_{2} \ldots, y_{n}\right)}{\prod_{i} y_{i} \prod_{i<j}\left(y_{i}+y_{j}\right)} \prod_{i=1}^{n} r\left(\theta_{i}\right) \prod_{i<j} f\left(\theta_{i}-\theta_{j}\right) f\left(\theta_{i}+\theta_{j}\right)
$$

where the $Q_{n}$ are symmetric polynomials of its variables, and the minimal one-particle form factor is given by

$$
r(\theta)=\frac{i \sinh \theta}{(\sinh \theta-i \sin \gamma)\left(\sinh \theta-i \sin \gamma^{\prime}\right)} u(\theta, B) \quad, \quad \gamma=\frac{\pi}{2}(E-1) \quad \gamma^{\prime}=\frac{\pi}{2}(F-1)
$$


with asymptotics $r(\theta) \sim 1$ when $\theta \rightarrow \infty$, where 1

$$
\begin{aligned}
u(\theta)= & \exp \left\{\int_{0}^{\infty} \frac{d t}{t}\left[\frac{1}{\sinh \frac{t}{2}}-2 \cosh \frac{t}{2} \cos \left[\left(\frac{i \pi}{2}-\theta\right) \frac{t}{\pi}\right]\right] \times\right. \\
& \left.\frac{\sinh \frac{x B}{4}+\sinh \left(1-\frac{B}{2}\right) \frac{x}{2}+\sinh \frac{x}{2}}{\sinh ^{2} t}\right\}
\end{aligned}
$$

and

$$
H_{n}=\left(\frac{4 \sin \pi B / 2}{f(i \pi)}\right)^{n / 2}
$$

is a convenient normalization factor. Using the results of [2, 3] it is easy to derive the recursion relations satisfied by the polynomials $Q_{n}$ :

$$
\begin{array}{ll}
\mathcal{K}: & Q_{2}(-y, y)=0 \\
& Q_{n+2}\left(-y, y, y_{1}, \ldots, y_{n}\right)= \\
& \left(y^{2}-4 \cos ^{2} \gamma\right)\left(y^{2}-4 \cos ^{2} \gamma^{\prime}\right) P_{n}\left(y \mid y_{1}, \ldots, y_{n}\right) Q_{n}\left(y_{1}, \ldots, y_{n}\right) \quad \text { for } n>0 \\
\mathcal{B}: \quad & Q_{1}(0)=0 \\
& Q_{n+1}\left(0, y_{1}, \ldots, y_{n}\right)= \\
& 4 \cos \gamma \cos \gamma^{\prime} B_{n}\left(y_{1}, \ldots, y_{n}\right) Q_{n}\left(y_{1}, \ldots, y_{n}\right) \quad \text { for } n>0
\end{array}
$$

where

$$
B_{n}\left(y_{1}, \ldots, y_{n}\right)=\frac{1}{4 \sin \frac{\pi B}{2}}\left(\prod_{i=1}^{n}\left(y_{i}-2 \sin \frac{\pi B}{2}\right)-\prod_{i=1}^{n}\left(y_{i}+2 \sin \frac{\pi B}{2}\right)\right)
$$

and

$$
P_{n}\left(y \mid y_{1}, \ldots y_{n}\right)=\frac{1}{2\left(y_{+}-y_{-}\right)}\left[\prod_{i=1}^{n}\left(y_{i}-y_{-}\right)\left(y_{i}+y_{+}\right)-\prod_{i=1}^{n}\left(y_{i}+y_{-}\right)\left(y_{i}-y_{+}\right)\right]
$$

with the notations

$$
\begin{aligned}
& y_{+}=\omega z+\omega^{-1} z^{-1} \\
& y_{-}=\omega^{-1} z+\omega z^{-1} \quad, \quad \omega=e^{i \pi \frac{B}{2}}
\end{aligned}
$$

with the auxiliary variable $z$ defined as a solution of $y=z+z^{-1}$ (i.e. writing $y=2 \cosh \theta$ one has $z=\mathrm{e}^{\theta}$ ).

The two-point functions can be computed from a spectral representation:

$$
\begin{aligned}
\rho_{\mathcal{A B}}(m t) & =\langle 0|\mathcal{A}(t) \mathcal{B}(0)| 0\rangle \\
& =\sum_{n=0}^{\infty} \frac{1}{(2 \pi)^{n}} \int_{\theta_{1}>\ldots>\theta_{n}>0} d \theta_{1} \ldots d \theta_{n} f_{n}\left(\theta_{1}, \ldots, \theta_{n}\right) \exp \left(-i m t \sum_{i=1}^{n} \cosh \theta_{i}\right)
\end{aligned}
$$

\footnotetext{
${ }^{1}$ Notice that the normalization used here differs from that in the earlier papers [2, 3].
} 
where time translation invariance was used and

$$
f_{n}\left(\theta_{1}, \ldots, \theta_{n}\right)=F_{n}^{\mathcal{A}}\left(\theta_{1}, \ldots, \theta_{n}\right)^{\dagger} F_{n}^{\mathcal{B}}\left(\theta_{1}, \ldots, \theta_{n}\right)
$$

The $f_{n}$ are symmetric (and also even) in all their variables, therefore the spectral expansion (3.9) can be written in the following form for the Euclidean two-point function

$$
\rho_{\mathcal{A} \mathcal{B}}(m \tau)=\sum_{n=0}^{\infty} \frac{1}{n !} \frac{1}{(2 \pi)^{n}} \int_{0}^{\infty} d \theta_{1} \int_{0}^{\infty} d \theta_{2} \ldots \int_{0}^{\infty} d \theta_{n} e^{-m \tau \sum_{i} \cosh \theta_{i}} f_{n}\left(\theta_{1}, \ldots, \theta_{n}\right)
$$

The operators of interest can be classified according to their scaling dimensions, which means that the two-point function must have a power-like short-distance singularity

$$
\rho_{\mathcal{A B}}(m \tau)=\frac{1}{\tau^{2 \Delta_{\mathcal{A B}}}}+\ldots
$$

where $\Delta_{\mathcal{A B}}$ is an exponent determined by the ultraviolet scaling weights of the local fields.

\subsection{The family of minimal solutions and the cumulant expansion}

In the earlier paper [3] it was shown that there exist an infinite family of solutions for which the polynomials $Q_{n}$ have the minimum possible degree

$$
\operatorname{deg} Q_{n}=\frac{n(n+1)}{2}
$$

These can be thought to correspond to the exponential operators

$$
\mathrm{e}^{\alpha \Phi(t, x=0)}
$$

of which only a countably infinite number is independent since they can all be expanded in terms of powers of the field:

$$
\mathrm{e}^{\alpha \Phi(t, 0)}=\sum_{k=1}^{\infty} \frac{\alpha^{k}}{k !} \Phi^{k}(t, 0)
$$

For any minimal solution the corresponding form factor has a finite limit when all rapidities are taken to infinity simultaneously

$$
F_{n}\left(\theta_{1}+\lambda, \theta_{2}+\lambda, \ldots, \theta_{n}+\lambda\right) \rightarrow \tilde{F}_{n}\left(\theta_{1}, \theta_{2}, \ldots, \theta_{n}\right)+O\left(\mathrm{e}^{-\lambda}\right)
$$

which means that every multi-particle contribution in the spectral expansion (3.9) individually behaves as

$$
\tau^{-2 \delta}
$$

with the naive scaling dimension $\delta$ equal to 0 [2, 3]. 
However, the true scaling dimension in general turns out to be different from 0 due to logarithmic corrections in the individual multi-particle contributions, which may sum up to give an anomalous dimension. It can be computed using the cumulant expansion of the logarithm of the two-point function [13] (for a very nice discussion see also [14]). Consider the conformal operator product expansion

$$
\mathcal{A}(\tau) \mathcal{B}(0) \sim \sum_{h_{i}} \frac{C_{\mathcal{A B}}^{i}}{\tau^{h_{\mathcal{A}}+h_{\mathcal{B}}-h_{i}}} \mathcal{O}_{i}(0)
$$

where $h_{\mathcal{A}}$ and $h_{\mathcal{B}}$ are the ultraviolet weights of the fields $\mathcal{A}$ and $\mathcal{B}$, while the $h_{i}$ are the weight of the $\mathcal{O}_{i}$. It is obvious that

$$
2 \Delta_{\mathcal{A B}}=h_{\mathcal{A}}+h_{\mathcal{B}}-h_{\min }
$$

where $h_{\text {min }}$ is the minimum of the weights $h_{i}$ of the operators $\mathcal{O}_{i}$ appearing in the expansion. Let us suppose that the limiting function (3.11) satisfies an asymptotic factorization property of the form

$$
\tilde{F}_{n}\left(\theta_{1}, \ldots, \theta_{k}, \theta_{k+1}+\lambda, \ldots, \theta_{n}+\lambda\right)=\tilde{F}_{k}\left(\theta_{1}, \ldots, \theta_{k}\right) \tilde{F}_{n-k}\left(\theta_{k+1}, \ldots, \theta_{n}\right)+O\left(\mathrm{e}^{-\lambda}\right)
$$

both for the form factors of $\mathcal{A}$ and $\mathcal{B}$. The leading $n=0$ term in the spectral expansions is a constant given by the vacuum expectation value of the field, which is assumed to be 1. In the case of bulk form factors (3.12) with the particular normalization given above entails that the operator has a unit vacuum expectation value [15]. For boundary form factors this can be carried over as a reasonable assumption which was used previously to normalize the minimal form factor of the boundary Lee-Yang model in 22. This assumption is reasonable from the consistency of the arguments in this paper and is also checked explicitly in subsection 4.2 .

Under the above assumptions, the logarithm of the correlation function can be written as

$$
\log \rho_{\mathcal{A B}}(m \tau)=\sum_{n=1}^{\infty} \frac{1}{n !} \frac{1}{(2 \pi)^{n}} \int_{0}^{\infty} d \theta_{1} \int_{0}^{\infty} d \theta_{2} \ldots \int_{0}^{\infty} d \theta_{n} \mathrm{e}^{-m \tau \sum_{i} \cosh \theta_{i}} c_{n}\left(\theta_{1}, \ldots, \theta_{n}\right)
$$

where the $c_{n}$ are the cumulants of the functions $f_{n}$ defined recursively by

$$
\begin{aligned}
& f_{1}\left(\theta_{1}\right)=c_{1}\left(\theta_{1}\right) \quad, \quad f_{2}\left(\theta_{1}, \theta_{2}\right)=c_{2}\left(\theta_{1}, \theta_{2}\right)+c_{1}\left(\theta_{1}\right) c_{1}\left(\theta_{2}\right) \\
& f_{3}\left(\theta_{1}, \theta_{2}, \theta_{3}\right)=c_{3}\left(\theta_{1}, \theta_{2}, \theta_{3}\right)+c_{1}\left(\theta_{1}\right) c_{2}\left(\theta_{2}, \theta_{3}\right)+c_{1}\left(\theta_{2}\right) c_{2}\left(\theta_{1}, \theta_{3}\right)+c_{1}\left(\theta_{3}\right) c_{2}\left(\theta_{1}, \theta_{2}\right) \\
& +c_{1}\left(\theta_{1}\right) c_{1}\left(\theta_{2}\right) c_{1}\left(\theta_{3}\right)
\end{aligned}
$$

Defining

$$
\tilde{c}_{n}\left(\theta_{1}, \ldots, \theta_{n}\right)=\lim _{\lambda \rightarrow \infty} c_{n}\left(\theta_{1}+\lambda, \ldots, \theta_{n}+\lambda\right)
$$


it is easy to see that the functions $\tilde{c}_{n}$ depend only on the differences of the rapidities. From (3.12) it is easy to obtain the following property of the asymptotic cumulants

$$
\tilde{c}_{n}\left(\theta_{1}, \ldots, \theta_{k}, \theta_{k+1}+\lambda, \ldots, \theta_{n}+\lambda\right) \sim O\left(\mathrm{e}^{-\lambda}\right) \quad k=1, \ldots, n-1
$$

and also note that

$$
c_{n}\left(\theta_{1}+\lambda, \ldots, \theta_{n}+\lambda\right)=\tilde{c}_{n}\left(\theta_{1}, \ldots, \theta_{n}\right)+O\left(\mathrm{e}^{-\lambda}\right)
$$

It can then be shown that

$$
2 \Delta_{\mathcal{A} B}=\sum_{n=1}^{\infty} \frac{1}{n !} \frac{1}{(2 \pi)^{n}} \int_{-\infty}^{\infty} d \theta_{2} \ldots \int_{-\infty}^{\infty} d \theta_{n} \tilde{c}_{n}\left(0, \theta_{2}, \ldots, \theta_{n}\right)
$$

The derivation of this result is a bit more involved than in the bulk case where the translational invariance of the form factor in rapidity space can be used. Let us examine the $n=1$ contribution in more detail. The formulae encountered will also be useful in subsection 4.2. Consider the integral

$$
\int_{0}^{\infty} \frac{d \theta}{2 \pi} c_{1}(\theta) \mathrm{e}^{-m \tau \cosh \theta}
$$

Due to (3.16), $c_{1}(\theta)$ approaches its limiting value exponentially fast

$$
c_{1}(\theta)=\tilde{c}_{1}+O\left(\mathrm{e}^{-\alpha \theta}\right)
$$

The exponential factor has the property

$$
\mathrm{e}^{-m \tau \cosh \theta} \sim 1 \quad \theta \ll \log \frac{2}{m \tau}
$$

and therefore in the limit of small $\tau$

$$
\int_{0}^{\infty} \frac{d \theta}{2 \pi} c_{1}(\theta) \mathrm{e}^{-m \tau \cosh \theta} \sim \int_{0}^{\infty} \frac{d \theta}{2 \pi}\left(c_{1}(\theta)-\tilde{c}_{1}\right)+\int_{0}^{\infty} \frac{d \theta}{2 \pi} \tilde{c}_{1} \mathrm{e}^{-m \tau \cosh \theta}
$$

which can be evaluated as

$$
\text { constant }+\frac{\tilde{c}_{1}}{2 \pi} K_{0}(m \tau)
$$

Using the asymptotics

$$
K_{0}(m \tau) \sim-\log m \tau+\log 2-\gamma_{E}
$$

(where $\gamma_{E}=0.577215 \ldots$ is Euler's constant) the short-distance exponent is given in this approximation as

$$
2 \Delta_{\mathcal{A B}}=\frac{\tilde{c}_{1}}{2 \pi}+\ldots
$$

Turning to $n>1$, the expansion $(3.13)$ can be rewritten as

$$
\log \rho_{\mathcal{A} \mathcal{B}}(m \tau)=\sum_{n=1}^{\infty} \frac{1}{n !} \frac{1}{(2 \pi)^{n}} \frac{1}{2^{n}} \int_{-\infty}^{\infty} d \theta_{1} \int_{-\infty}^{\infty} d \theta_{2} \ldots \int_{-\infty}^{\infty} d \theta_{n} \mathrm{e}^{-m \tau \sum_{i} \cosh \theta_{i}} c_{n}\left(\theta_{1}, \ldots, \theta_{n}\right)
$$


using that the functions $f_{n}$ and consequently also $c_{n}$ are even in all their arguments. For $m \tau \ll 1$, the support of the integrand is concentrated inside the hypercube

$$
\left|\theta_{i}\right| \lesssim \log (2 / m \tau)
$$

and in the interior (except for a transient shell-like region whose thickness is $O(1)$ independent of $\tau$ )

$$
\mathrm{e}^{-m \tau \sum_{i} \cosh \theta_{i}} \sim 1
$$

Using the properties (3.15) and (3.16) $c_{n}$ can be replaced by $\tilde{c}_{n}$ up to $O(1)$ terms. One can now repeat the same procedure in the $\theta_{1}$ integral as for $n=1$, following the line of the derivation used in the bulk case [14]. However, some additional care must be taken since the functions $c_{n}$ are even, and therefore there are $2^{n-1}$ independent asymptotic directions in which the integrand is non-vanishing:

$$
c_{n}\left(\theta_{1}+\lambda, \theta_{2}+\sigma_{2} \lambda, \ldots, \theta_{n}+\sigma_{n} \lambda\right) \rightarrow \tilde{c}_{n}\left(\theta_{1}, \sigma_{2} \theta_{2}, \ldots, \sigma_{n} \theta_{n}\right)
$$

depending on the choices of the signs $\sigma_{i}= \pm 1$ (the sign of $\theta_{1}$ is fixed as only the relative signs matter). After a redefinition of integration variables $\theta_{i} \rightarrow \sigma_{i} \theta_{i}$ this results in $2^{n-1}$ identical contribution which cancel out $n-1$ factors of $1 / 2$. The remaining factor $1 / 2$ can be used to map back the $\theta_{1}$ integration to the half-line where (3.19) can be used.

In the bulk the factor $1 / 2^{n}$ are absent, and translational invariance of the form factor of a spinless operator implies that $c_{n} \equiv \tilde{c}_{n}$. Due to (3.15) only the asymptotic region with all signs positive contributes, so there is no factor $2^{n-1}$ either. Therefore the bulk singularity exponent is twice the one in the boundary case (provided that the asymptotic cumulants are taken to be identical). However, if the singularity of the bulk two-point function is taken to be of the form

$$
\frac{1}{\tau^{4 \Delta_{\mathcal{A B}}}}
$$

then this factor cancels out in the final formula for $\Delta_{\mathcal{A B}}$ and so the bulk cumulant expansion is identical to (3.17). The additional factor of 2 in the exponent is natural since in the bulk the conformal weight receives two identical contributions from the left and the right movers.

\subsection{The limiting case: bulk form factor solutions in the sinh- Gordon model}

Now the task is to resolve the ambiguity in the minimal solution. From [3] it is known to originate from the kernel of the recursion relations, which is a homogeneous polynomial of order $n(n+1) / 2$ at $n$-particle level. Let us introduce

$$
\tilde{Q}_{n}\left(y_{1}, \ldots, y_{n}\right)=\lim _{\Lambda \rightarrow \infty} \Lambda^{-n(n+1) / 2} Q_{n}\left(\Lambda y_{1}, \ldots, \Lambda y_{n}\right)
$$

which gives the terms in $Q_{n}$ which are exactly of degree $n(n+1) / 2$ (all other terms have lower degree). 
Taking now a solution of the form (3.2), the limiting procedure (3.11) gives

$$
\begin{aligned}
\tilde{F}_{n}\left(\theta_{1}, \theta_{2}, \ldots, \theta_{n}\right) & =\lim _{\lambda \rightarrow \infty} F_{n}\left(\theta_{1}+\lambda, \theta_{2}+\lambda, \ldots, \theta_{n}+\lambda\right) \\
& =H_{n} \frac{\tilde{Q}_{n}\left(x_{1}, x_{2} \ldots, x_{n}\right)}{\prod_{i} x_{i} \prod_{i<j}\left(x_{i}+x_{j}\right)} \prod_{i<j} f\left(\theta_{i}-\theta_{j}\right)
\end{aligned}
$$

where $x_{i}=\mathrm{e}^{\theta_{i}}$. It was observed in [3] that as a consequence of the boundary form factor axioms listed in subsection 3.1, $\tilde{F}$ is always a solution of the bulk form factor axioms built upon the same bulk $S$ matrix. For the bulk sinh-Gordon theory, a complete set of minimal solutions was found by Koubek and Mussardo in [16]. Let us define the elementary symmetric polynomials by

$$
\begin{aligned}
\prod_{i=1}^{n}\left(x+x_{i}\right)= & \sum_{l=1}^{n} x^{n-l} \sigma_{l}^{(n)}\left(x_{1}, \ldots, x_{n}\right) \\
\sigma_{l}^{(n)} \equiv 0 & \text { if } l<0 \text { or } l>n
\end{aligned}
$$

The upper index will be omitted in the sequel, as the number of variables will always be clear from the context. Let us also denote

$$
[n]=\frac{\omega^{n}-\omega^{-n}}{\omega-\omega^{-1}}=\frac{\sin \frac{n \pi B}{2}}{\sin \frac{\pi B}{2}}
$$

Koubek and Mussardo state that the following family of form factor solutions parametrized by the real number $k$

$$
\tilde{F}_{n}^{(k)}\left(\theta_{1}, \theta_{2}, \ldots, \theta_{n}\right)=H_{n} \frac{P_{n}^{(k)}\left(x_{1}, x_{2} \ldots, x_{n}\right)}{\prod_{i<j}\left(x_{i}+x_{j}\right)} \prod_{i<j} f\left(\theta_{i}-\theta_{j}\right)
$$

where the polynomials $P_{n}^{(k)}$ are given by

$$
\begin{aligned}
P_{1}^{(k)}= & {[k] } \\
P_{n}^{(k)}= & {[k] \operatorname{det} M^{(n)}(k) \quad n>1 } \\
& M_{i j}^{(n)}(k)=[i-j+k] \sigma_{2 i-j}\left(x_{1}, x_{2} \ldots, x_{n}\right) \quad i, j=1, \ldots, n-1
\end{aligned}
$$

correspond to the bulk exponential operators

$$
\mathrm{e}^{k g \Phi}
$$

normalized so that their vacuum expectation value is unity:

$$
\left\langle\mathrm{e}^{k g \Phi}\right\rangle=1
$$


They support the identification as follows. The above solutions satisfy the asymptotic factorization property (3.12), derived in [15] for form factors of relevant primary fields. In addition, their conformal weights to lowest order in $g^{2}$ (computed from the two-point function) are given by

$$
\Delta_{k}=\bar{\Delta}_{k}=-\frac{k^{2} g^{2}}{8 \pi}
$$

which equal the conformal weights of the exponential operator $\mathrm{e}^{k g \Phi}$ computed in the free boson conformal field theory. In section 4 of the present paper more supporting evidence is provided for this identification.

\subsection{Resolution of the ambiguity and explicit form factor solutions}

Based on the preceding considerations the ambiguity in the boundary form factor solution can be fixed by requiring that

$$
\tilde{Q}_{n}=P_{n}^{(k)} \sigma_{n}
$$

i.e.

$$
\begin{aligned}
Q_{n}\left(y_{1}, \ldots, y_{n}\right)= & P_{n}^{(k)}\left(y_{1}, \ldots, y_{n}\right) \sigma_{n}\left(y_{1}, \ldots, y_{n}\right) \\
& + \text { terms of degree strictly less than } n(n+1) / 2
\end{aligned}
$$

For such a form factor the limiting procedure (3.11) gives $\tilde{F}_{n}^{(k)}$. Therefore the limiting two-point function cumulants defined in (3.14) will be the same as for the bulk operator $\mathrm{e}^{k g \Phi}$. By comparing the cumulant expansion (3.17) to its bulk counterpart, this means that the boundary operator corresponding to this solution has the conformal weight

$$
h_{k}=-\frac{k^{2} g^{2}}{8 \pi}
$$

(at least to lowest order in $g^{2}$ ).

The boundary exponential field $\mathrm{e}^{\alpha \Phi(t, 0)}$ has conformal weight

$$
-\frac{\alpha^{2}}{2 \pi}
$$

which leads to the conjecture that the boundary form factors satisfying (3.21) correspond to the operators

$$
\mathrm{e}^{\frac{k g}{2} \Phi}
$$

The factor of one-half in the exponent is consistent with the form of the boundary perturbation in the action (2.3), where a bulk perturbation which is a combination of $\mathrm{e}^{ \pm g \Phi}$ is accompanied by a boundary perturbation which is a combination of $\mathrm{e}^{ \pm g \Phi / 2}$.

The recurrence relations (3.5) can be solved as follows. The one-particle form factor is uniquely given by

$$
Q_{1}^{(k)}\left(y_{1}\right)=P_{1}^{(k)} \sigma_{1}\left(y_{1}\right)=[k] y_{1}
$$


The boundary kinematical recursion coefficient (3.6) can be expressed in terms of the elementary symmetric polynomials $\sigma_{l}$ in the form

$$
B_{n}\left(y_{1}, \ldots, y_{n}\right)=-\sum_{l=0}^{\left[\frac{n-1}{2}\right]}\left(2 \cos \frac{\pi B}{2}\right)^{2 l} \sigma_{n-1-2 l}
$$

Supposing that the solution up to $n-1$ particle level is known, one can write the following Ansatz for the $n$-particle form factor

$$
Q_{n}^{(k)}=\epsilon_{1} \epsilon_{2} B_{n-1} Q_{n-1}^{(k)}+\sigma_{n} P_{n}^{(k)}+\sigma_{n} A_{n}^{(k)}
$$

where $A_{n}^{(k)}$ is a general linear combination of products of $\sigma_{l}$ with total degree strictly less than $n(n-1) / 2$, and the first term is understood with the replacement

$$
\sigma_{l}^{(n-1)} \rightarrow \sigma_{l}^{(n)}
$$

and the notation

$$
\epsilon_{1}=2 \cos \gamma \quad, \quad \epsilon_{2}=2 \cos \gamma^{\prime}
$$

was introduced. The Ansatz (3.22) automatically solves the boundary kinematical recursion $\mathcal{B}$ in (3.5) and therefore the coefficients of $A_{n}^{(k)}$ can be determined from the bulk kinematical recursion $\mathcal{K}$. Since the kernel of the recursion is already fixed by (3.22), the solution is unique. It is given by

$$
\begin{aligned}
& A_{2}^{(k)}=0 \\
& A_{3}^{(k)}=[k]\left(\epsilon_{1}^{2}+\epsilon_{2}^{2}-[k] \epsilon_{1} \epsilon_{2}\right) \sigma_{1} \\
& A_{4}^{(k)}=4 \sin ^{2} \frac{\pi B}{2}[k] \sigma_{1} \sigma_{3}+[k]^{2}\left(\epsilon_{1}^{2}+\epsilon_{2}^{2}-[k] \epsilon_{1} \epsilon_{2}\right) \sigma_{1}^{2}\left(\sigma_{2}+4 \sin ^{2} \frac{\pi B}{2}\right)
\end{aligned}
$$

up to 4-particle level, and it can easily be extended to higher levels using any symbolic algebra software.

\section{Evaluating the cumulant expansion}

To provide evidence that the solution indeed corresponds to the exponential operators as stated, let us study the short-distance behaviour of the two-point correlators arising from it. It was already mentioned that Koubek and Mussardo calculated the ultraviolet scaling dimension to order $g^{2}$ by studying the short-distance limit of the two-point function [16]; Babujian and Karowski evaluated it from the cumulant expansion up to two-particle level [14. Here the most singular term in the short-distance operator product of any two of the operators in the family of minimal solutions is expanded in powers of $g^{2}$ to order $g^{6}$. In addition, the normalization of these operators is also evaluated to order $g^{2}$. 
The conformal operator product algebra gives the leading term in the short-distance limit of the two-point function as

$$
\left\langle 0\left|\mathrm{e}^{k \frac{g}{2} \Phi(\tau)} \mathrm{e}^{l \frac{g}{2} \Phi(0)}\right| 0\right\rangle \sim \frac{1}{\tau^{2 \Delta_{k l}}}\left\langle 0\left|\mathrm{e}^{(k+l) \frac{g}{2} \Phi(0)}\right| 0\right\rangle+\ldots
$$

with

$$
2 \Delta_{k l}=-\frac{k^{2} g^{2}}{8 \pi}-\frac{l^{2} g^{2}}{8 \pi}+\frac{(k+l)^{2} g^{2}}{8 \pi}=\frac{k l g^{2}}{4 \pi}
$$

where the standard conformal normalization for the exponential operators was assumed. Recall that the operator thought to correspond to the form factor solution in the previous section is normalized to have unit vacuum expectation value, i.e. it takes the form

$$
V_{k}(\tau)=\frac{1}{\left\langle 0\left|\mathrm{e}^{k \frac{g}{2} \Phi(\tau)}\right| 0\right\rangle} \mathrm{e}^{k \frac{g}{2} \Phi(\tau)}
$$

so the short-distance expansion can be written as

$$
\left\langle 0\left|V_{k} V_{l}\right| 0\right\rangle \sim \frac{C_{k l}^{k+l}}{(m \tau)^{2 \Delta_{k l}}}\left\langle 0\left|V_{k+l}\right| 0\right\rangle+\ldots
$$

where the structure constant $C_{k l}^{k+l}$ is given by the ratio of the appropriate vacuum expectation values.

The cumulant expansion can be used to evaluate both $\Delta_{k l}$ and $C_{k l}^{k+l}$ [14. The first one can be expressed in terms the asymptotic cumulants $\tilde{c}_{k}$, and as a result the calculation is valid both for the boundary and the bulk case, since the asymptotic cumulants $\tilde{c}_{k}$ are the same. This is due to the way the ambiguity in the boundary solution was fixed using the bulk solution (see eqn. (3.21)).

On the other hand, the evaluation of $C_{k l}^{k+l}$ involves the full cumulants $c_{k}$. In the bulk theory these are identical to $\tilde{c}_{k}$, but for the boundary theory they differ from them. This is consistent with the fact that the $\tilde{c}_{k}$ are independent of the boundary parameters, while the vacuum expectation values of boundary fields depend on them in a very nontrivial way; therefore the appearance the full cumulant $c_{k}$ is reassuring. Below it is demonstrated that to order $g^{2}$ the result from the cumulant expansion agrees with the exact vacuum expectation values conjectured for the bulk theory in [17] and for the boundary case in [18].

\subsection{Evaluation of $\Delta_{k l}$}

Using the cumulant expansion (3.17), $\Delta_{k l}$ can be evaluated in a systematic expansion in powers of $g^{2}$. The $\tilde{c}_{n}$ can be obtained as cumulants of the asymptotic spectral functions

$$
\tilde{f}_{n}^{k l}\left(\theta_{1}, \ldots, \theta_{n}\right)=\lim _{\lambda \rightarrow \infty} f_{n}^{k l}\left(\theta_{1}+\lambda, \ldots, \theta_{n}+\lambda\right)
$$


which take the explicit form

$$
\tilde{f}_{n}^{k l}\left(\theta_{1}, \ldots, \theta_{n}\right)=H_{n}^{2} P_{n}^{(k)}\left(x_{1}, x_{2} \ldots, x_{n}\right) P_{n}^{(l)}\left(x_{1}, x_{2} \ldots, x_{n}\right) \prod_{i<j} \frac{\left|f\left(\theta_{i}-\theta_{j}\right)\right|^{2}}{\left(x_{i}+x_{j}\right)^{2}} \quad, \quad x_{i}=\mathrm{e}^{\theta_{i}}
$$

From (2.1) it is obvious that $f(\theta)=1+O\left(g^{2}\right)$ for any fixed $\theta \neq 0$, and the leading term of the polynomials $P_{n}^{(k)}$ is given by

$$
\begin{aligned}
P_{n}^{(k)}= & k \operatorname{det} m^{(n)}(k)+O\left(g^{2}\right) \\
\text { where } \quad & m_{i j}^{(n)}(k)=(i-j+k) \sigma_{2 i-j}\left(x_{1}, x_{2} \ldots, x_{n}\right) \quad i, j=1, \ldots, n-1
\end{aligned}
$$

while

$$
H_{n}=\left(\frac{4 \sin \pi B / 2}{f(i \pi)}\right)^{n / 2} \sim\left(\frac{g^{2}}{2}\right)^{n / 2}+O\left(g^{n+1}\right)
$$

As a result

$$
\tilde{f}_{n}^{k l} \sim g^{2 n} \Rightarrow \tilde{c}_{n}^{k l} \sim g^{2 n}
$$

so an expansion up to order $g^{2 n}$ only requires collecting the appropriate terms from cumulants with up to $n$ particles. The first cumulant can be easily expanded as

$$
\tilde{c}_{1}=H_{1}^{2}[k]^{2} \sim \frac{g^{2}}{2} k l+g^{6}\left(\frac{k l}{3072}-\frac{k l}{256 \pi}-\frac{k^{3} l+k l^{3}}{768}\right)+O\left(g^{10}\right)
$$

and using (3.20) gives

$$
2 \Delta_{k l} \sim \frac{g^{2}}{4 \pi} k l+O\left(g^{6}\right)
$$

Therefore to recover the intended result it must be shown that all higher order contributions cancel, which is demonstrated below up to order $g^{6}$.

At order $g^{4}$ the second asymptotic cumulant is needed. It takes the form

$$
\tilde{c}_{2}\left(\theta_{1}, \theta_{2}\right)=\frac{1}{4} g^{4} k^{2} l^{2}\left(\left|f\left(\theta_{1}-\theta_{2}\right)^{2}\right|-1\right)
$$

At first this seems to be of order $O\left(g^{6}\right)$, but some care must be taken. Although it is true that $f(\theta)=1+O\left(g^{2}\right)$ for any fixed $\theta \neq 0$, it also holds that $f(0)=0$. Therefore the expansion of $f(\theta)$ in powers of $g^{2}$ does not converge uniformly in the vicinity of the origin, and the series expansion cannot be exchanged with the integration. However, the required integral can be evaluated exactly [14]; it has the following small coupling expansion 2 ]

$$
\int_{0}^{\infty} d \theta\left(\left|f(\theta)^{2}\right|-1\right)=-\frac{\pi^{2}}{24} B^{2}+O\left(B^{3}\right)=O\left(g^{4}\right)
$$

and therefore the two-particle contribution is actually of order $O\left(g^{8}\right)$.

\footnotetext{
${ }^{2}$ In the formula derived in [14] there seems to be an overall sign mistake, which is corrected here.
} 
The third cumulant can be written as

$$
\tilde{c}_{3}\left(\theta_{1}, \theta_{2}, \theta_{3}\right)=\frac{g^{6}}{8}\left(k^{3} l+k l^{3}\right) \frac{\sigma_{3}}{\sigma_{3}-\sigma_{1} \sigma_{2}}+\frac{g^{6}}{8} k l\left(\frac{\sigma_{3}}{\sigma_{3}-\sigma_{1} \sigma_{2}}\right)^{2}+O\left(g^{8}\right)
$$

where the $\sigma_{k}$ are elementary symmetric polynomials of the variables $x_{i}=\mathrm{e}^{\theta_{i}}$. The integral can be evaluated as

$$
\frac{1}{6} \int_{-\infty}^{\infty} \frac{d \theta_{1}}{2 \pi} \int_{-\infty}^{\infty} \frac{d \theta_{2}}{2 \pi} \tilde{c}_{3}\left(\theta_{1}, \theta_{2}, \theta_{3}\right)=g^{6}\left(\frac{k^{3} l+k l^{3}}{768}+\frac{k l}{256 \pi}-\frac{k l}{3072}\right)
$$

which indeed cancels with the $g^{6}$ term in (4.2).

\subsection{Evaluation of $C_{k l}^{k+l}$}

$C_{k l}^{k+l}$ is the constant contribution to the logarithm of the two-point function in the shortdistance limit. It can be expressed as

$$
C_{k l}^{k+l}=\frac{\mathcal{G}(k+l, g)}{\mathcal{G}(k, g) \mathcal{G}(l, g)}
$$

where $\mathcal{G}(k, g)$ denotes the exact vacuum expectation value computed in [17]:

$$
\begin{aligned}
\mathcal{G}(k, g)=m^{2 a^{2}}\left\langle\mathrm{e}^{a \varphi}\right\rangle= & {\left[\frac{\Gamma\left[\frac{1}{2+2 b^{2}}\right] \Gamma\left[1+\frac{b^{2}}{2+2 b^{2}}\right]}{4 \sqrt{\pi}}\right]^{-2 a^{2}} \times } \\
& \exp \left\{\int_{0}^{\infty} \frac{d t}{t}\left[-\frac{\sinh ^{2}(2 a b t)}{2 \sinh ^{2}(b t) \sinh (t) \cosh \left(\left(1+b^{2}\right) t\right)}+2 a^{2} \mathrm{e}^{-2 t}\right]\right\}
\end{aligned}
$$

and the parameters can be expressed in our notations as

$$
b=\frac{g}{\sqrt{8 \pi}} \quad, \quad a=\frac{k g}{\sqrt{8 \pi}}
$$

For small $g$ it can be written as

$$
\mathcal{G}(k, g) \sim \exp \left(-\frac{k^{2} g^{2}}{4 \pi}\left(-\log 2+\gamma_{E}\right)\right)
$$

and so

$$
C_{k l}^{k+l} \sim \exp \left(-\frac{k l g^{2}}{2 \pi}\left(-\log 2+\gamma_{E}\right)\right)
$$

On the other hand, it can easily be evaluated from the cumulant expansion in the oneparticle approximation. For the bulk theory, the first term in (3.18) vanishes exactly and $\mathrm{sd}^{3}$

$$
\log C_{k l}^{k+l}=2 \frac{\tilde{c}_{1}}{2 \pi}\left(\log 2-\gamma_{E}\right)+\ldots=\frac{k l g^{2}}{2 \pi}\left(\log 2-\gamma_{E}\right)+O\left(g^{4}\right)
$$

\footnotetext{
${ }^{3} \mathrm{~A}$ factor of two was inserted to compensate for the difference between the bulk and boundary rapidity integrals.
} 
which agrees perfectly with (4.3).

Turning now to the boundary case, the exact vacuum expectation values of boundary fields in sine-Gordon theory were conjectured in [18]. With the following action

$$
\mathcal{A}_{\mathrm{bsG}}=\int d^{2} x\left[\frac{1}{4 \pi}\left(\partial_{t} \phi\right)^{2}-\frac{1}{4 \pi}\left(\partial_{x} \phi\right)^{2}-2 \mu \cos 2 \beta \phi\right]-2 \mu_{B} \int d t \cos \beta\left(\phi(t, 0)-\phi_{0}\right)
$$

the boundary one-point functions can be written in the following form:

$$
\left\langle\mathrm{e}^{i a \phi(t, 0)}\right\rangle=\left(\frac{\pi \mu}{\Gamma\left(\beta^{2}\right)}\right)^{\frac{a^{2}}{2\left(1-\beta^{2}\right)}} g_{0}(a, \beta) g_{S}(a, \beta) g_{A}(a, \beta)
$$

where 4

$$
\begin{aligned}
& g_{0}(a, \beta)=\exp \left\{\int_{0}^{\infty} \frac{d t}{t}\left[\frac{2 \sinh ^{2}(a \beta t)\left(\mathrm{e}^{\left(1-\beta^{2}\right) t / 2} \cosh (t / 2) \cosh \left(\beta^{2} t / 2\right)-1\right)}{\sinh \left(\beta^{2} t\right) \sinh (t) \sinh \left(\left(1-\beta^{2}\right) t\right)}-a^{2} \mathrm{e}^{-t}\right]\right\} \\
& g_{S}(a, \beta)=\exp \left\{\int_{0}^{\infty} \frac{d t}{t} \frac{\sinh ^{2}(a \beta t)(2-\cos (2 z t)-\cos (2 \bar{z} t))}{2 \sinh \left(\beta^{2} t\right) \sinh (t) \sinh \left(\left(1-\beta^{2}\right) t\right)}\right\} \\
& g_{A}(a, \beta)=\exp \left\{\int_{0}^{\infty} \frac{d t}{t} \frac{\sinh (2 a \beta t)(\cos (2 z t)-\cos (2 \bar{z} t))}{\sinh \left(\beta^{2} t\right) \sinh (t) \cosh \left(\left(1-\beta^{2}\right) t\right)}\right\}
\end{aligned}
$$

and 5

$$
\cosh ^{2} \pi z=\mathrm{e}^{-2 i \beta \phi_{0}} \frac{\mu_{B}^{2} \sin \pi \beta^{2}}{\mu} \quad, \quad \cosh ^{2} \pi \bar{z}=\mathrm{e}^{2 i \beta \phi_{0}} \frac{\mu_{B}^{2} \sin \pi \beta^{2}}{\mu}
$$

provided the operators are normalized as

$$
\begin{aligned}
\mathrm{e}^{2 i a \phi(x)} \mathrm{e}^{-2 i a \phi(x)} & \sim \frac{1}{|x-y|^{4 a^{2}}}+\ldots \\
\mathrm{e}^{i a \phi(t, 0)} \mathrm{e}^{-i a \phi\left(t^{\prime}, 0\right)} & \sim \frac{1}{\left|t-t^{\prime}\right|^{2 a^{2}}}+\ldots
\end{aligned}
$$

The coupling $\mu$ can be related to the mass $m$ of the lightest breather as follows [19]:

$$
\mu=\frac{\Gamma\left(\beta^{2}\right)}{\pi \Gamma\left(1-\beta^{2}\right)}\left[\frac{m}{2 \sin \frac{\pi \beta^{2}}{2\left(1-\beta^{2}\right)}} \frac{\sqrt{\pi} \Gamma\left(\frac{1}{2\left(1-\beta^{2}\right)}\right)}{2 \Gamma\left(\frac{\beta^{2}}{2\left(1-\beta^{2}\right)}\right)}\right]^{2-2 \beta^{2}}
$$

\footnotetext{
${ }^{4}$ The integral in the exponent of $g_{S}$ contains an additional factor of $1 / 2$ compared to the formula in [18. The need for such a factor was already noted in [20], and it was also confirmed in a private discussion with Al.B. Zamolodchikov.

${ }^{5}$ In sine-Gordon theory, $\bar{z}$ is the complex conjugate of $z$, but this does not remain true under analytic continuation to sinh-Gordon theory.
} 
Eqn. (4.5) can be expanded for small $\beta$ as

$$
\mathcal{G}_{B}(\kappa, \beta)=m^{-2 \kappa^{2} \beta^{2}}\left\langle\mathrm{e}^{i \kappa \beta \phi(t, 0)}\right\rangle \sim \mathcal{N}(\kappa, \beta) \bar{g}_{0}(\kappa, \beta) \bar{g}_{S}(\kappa, \beta) \bar{g}_{A}(\kappa, \beta)
$$

with

$$
\begin{aligned}
\mathcal{N}(\kappa, \beta) & =1-2 \kappa^{2} \beta^{2} \log 2+O\left(\beta^{4}\right) \\
\bar{g}_{0}(\kappa, \beta) & =1+\kappa^{2} \beta^{2}\left(1+\gamma_{E}+\log 2\right)+O\left(\beta^{4}\right) \\
\bar{g}_{S}(\kappa, \beta) & =1+\kappa^{2} \beta^{2}\left(\frac{1}{2} \pi z \operatorname{coth} \pi z+\frac{1}{2} \pi \bar{z} \operatorname{coth} \pi \bar{z}-1\right)+O\left(\beta^{4}\right) \\
\bar{g}_{A}(\kappa, \beta) & =1+\kappa \int_{0}^{\infty} \frac{d t}{t}(\cos (2 z t)-\cos (2 \bar{z} t))\left(\frac{4}{\sinh [2 t]}+\frac{2 t}{\cosh [t]^{2}} \beta^{2}\right)+O\left(\beta^{4}\right)
\end{aligned}
$$

Due to linearity of $\bar{g}_{A}$ in $\kappa$ its contribution drops from $C_{k l}^{k+l}$, so it is not necessary to evaluate it explicitly. The sinh-Gordon expectation values can be obtained by taking the analytic continuation $\beta \rightarrow i g / \sqrt{8 \pi}$, under which the operator

$$
\mathrm{e}^{k \frac{g}{2} \Phi(\tau)}
$$

corresponds to putting $\kappa=k$. Collecting all the terms the final expression is

$$
\begin{aligned}
\log C_{k l}^{k+l} & =\left.\log \frac{\mathcal{G}_{B}(k+l, \beta)}{\mathcal{G}_{B}(k, \beta) \mathcal{G}_{B}(l, \beta)}\right|_{\beta \rightarrow i g / \sqrt{8 \pi}} \\
& =-\frac{k l g^{2}}{4 \pi}\left(-\log 2+\gamma_{E}+\frac{1}{2} \pi z \operatorname{coth} \pi z+\frac{1}{2} \pi \bar{z} \operatorname{coth} \pi \bar{z}\right)+O\left(g^{4}\right)
\end{aligned}
$$

On the other hand, from the cumulant expansion

$$
\log C_{k l}^{k+l}=\frac{\tilde{c}_{1}}{2 \pi}\left(\log 2-\gamma_{E}\right)+\int_{0}^{\infty} \frac{d \theta}{2 \pi}\left(c_{1}(\theta)-\tilde{c}_{1}\right)
$$

From (3.3) it is easy to obtain that

$$
r(\theta)=\frac{i \sinh \theta(1-i \sinh \theta)}{(\sinh \theta-i \sin \gamma)\left(\sinh \theta-i \sin \gamma^{\prime}\right)}+O\left(g^{2}\right)
$$

and so

$$
\int_{0}^{\infty} \frac{d \theta}{2 \pi}\left(c_{1}(\theta)-\tilde{c}_{1}\right)=\frac{k l g^{2}}{4 \pi} \int_{0}^{\infty} d \theta\left\{\frac{\sinh ^{2} \theta\left(1+\sinh ^{2} \theta\right)}{\left(\sinh ^{2} \theta+\sin ^{2} \gamma\right)\left(\sinh ^{2} \theta+\sin ^{2} \gamma^{\prime}\right)}-1\right\}
$$

The integral can be evaluated using

$$
\int_{0}^{\infty} \frac{d \theta}{\sinh ^{2} \theta+\sin ^{2} \gamma}=\frac{\pi-2|\gamma|}{|\sin 2 \gamma|} \quad \text { for } \quad-\frac{\pi}{2}<\gamma<\frac{\pi}{2}
$$


For definiteness let us suppose that

$$
-\frac{\pi}{2}<\gamma, \gamma^{\prime}<0
$$

which means that $0<E, F<1$ as a consequence of the parameter identification in (3.3). This leads to

$$
\begin{aligned}
\log C_{k l}^{k+l}= & \frac{k l g^{2}}{4 \pi}\left[\log 2-\gamma_{E}-\frac{1}{2}\left(\pi+\gamma+\gamma^{\prime}\right) \cot \left(\pi+\gamma+\gamma^{\prime}\right)\right. \\
& \left.-\frac{1}{2}\left(\gamma-\gamma^{\prime}\right) \cot \left(\gamma-\gamma^{\prime}\right)\right] \\
= & \frac{k l g^{2}}{4 \pi}\left[\log 2-\gamma_{E}-\frac{1}{4} \pi(E+F) \cot \frac{1}{2} \pi(E+F)\right. \\
& \left.-\frac{1}{4} \pi(E-F) \cot \frac{1}{2} \pi(E-F)\right]
\end{aligned}
$$

The expressions (4.7) and (4.8) match provided

$$
z=\frac{1}{2}(E-F) \quad, \quad \bar{z}=\frac{1}{2}(E+F)
$$

Some care must be taken because there is a branch choice ambiguity in the $z, \bar{z}$ parametrization which is obvious from (4.6); in (4.9) an implicit choice was made. It is only necessary to show that there exists one particular choice which is consistent; the correspondence on the other branches (i.e. outside the region $0<E, F<1$ ) can be obtained by analytic continuation.

The consistency condition is that (4.9) must provide an identification of the reflection factor

$$
R(\theta)=\left(\frac{1}{2}\right)_{\theta}\left(\frac{1}{2}+\frac{B}{4}\right)_{\theta}\left(1-\frac{B}{4}\right)_{\theta}\left[\frac{E-1}{2}\right]_{\theta}\left[\frac{F-1}{2}\right]_{\theta}
$$

(cf. (2.4) ) of the sinh-Gordon particle to the that of the first sine-Gordon breather under the analytic continuation $\beta \rightarrow i g / \sqrt{8 \pi}$. The reflection factor of the first breather can be written as [9]

$$
\begin{aligned}
& R^{(1)}(\vartheta)=\left(\frac{1}{2}\right)_{\theta}\left(\frac{1}{2}-\frac{\xi}{2}\right)_{\theta}\left(1+\frac{\xi}{2}\right)_{\theta}\left[\frac{\xi \eta}{\pi}-\frac{1}{2}\right]_{\theta}\left[\frac{i \xi \vartheta}{\pi}-\frac{1}{2}\right]_{\theta} \\
& \xi=\frac{\beta^{2}}{1-\beta^{2}}
\end{aligned}
$$

which can be compared with (2.4). Under the analytic continuation $\beta \rightarrow i g / \sqrt{8 \pi}$

$$
B=-2 \xi
$$


and so the factors independent of the boundary condition agree. From the following relation between the Lagrangian and bootstrap parameters

$$
\begin{aligned}
\cos \left(\beta^{2} \eta\right) \cosh \left(\beta^{2} \vartheta\right) & =\mu_{B} \sqrt{\frac{\sin \pi \beta^{2}}{\mu}} \cos \left(\beta \phi_{0}\right) \\
\sin \left(\beta^{2} \eta\right) \sinh \left(\beta^{2} \vartheta\right) & =\mu_{B} \sqrt{\frac{\sin \pi \beta^{2}}{\mu}} \sin \left(\beta \phi_{0}\right)
\end{aligned}
$$

derived by Al.B. Zamolodchikov [11] (see also 20]), comparing to (4.6) the following relation is obtained:

$$
z=\frac{\beta^{2}}{\pi}(\eta-i \vartheta) \quad, \quad \bar{z}=\frac{\beta^{2}}{\pi}(\eta+i \vartheta)
$$

where an appropriate choice of branches is understood. To the leading order 6 in $\beta^{2}(4.9)$ and (4.12) are equivalent to

$$
E=\frac{2 \xi \eta}{\pi} \quad, \quad F=\frac{2 i \xi \vartheta}{\pi}
$$

which makes the reflection factors (4.10) and (4.11) identical.

\section{Discussion and outlook}

With the method developed in this paper, it proved possible to organize the infinitely many minimal boundary form factor solutions of sinh-Gordon theory encountered in [3] into well-defined families by restricting the highest-growing term to be equal to the bulk form factor of a given exponential operator. It was shown that such a solution has the correct scaling dimension to order $g^{6}$, and the claim that its vacuum expectation value is normalized to 1 matches with the exact vacuum expectation values conjectured in [18] to order $g^{2}$.

The restriction leading to unique determination of the solution was made possible by the observation made in [3] that the dominant asymptotic contribution of the boundary form factor always solves the bulk form factor equations. It is very important to note that the construction always guarantees that the cumulant expansion for the scaling dimension of the boundary operator coincides with that of the bulk one which was used as an input for the solution of the boundary form factor bootstrap axioms.

This observation leads to a proposal for constructing form factors of relevant boundary operators. In the bulk, form factors of spinless relevant fields can be selected from the space of solutions to the form factor axioms by the asymptotic factorization condition (3.12) which was derived in [15]. Using these solutions as an input one can then construct solutions for relevant boundary fields whose scaling dimension coincides with the right (or equally, the left) conformal dimension of the corresponding bulk field. In fact, the form

\footnotetext{
${ }^{6}$ Since eqn. (4.9) itself is only valid to leading order in $\beta^{2}$, it doesn't make sense to take the comparison beyond this approximation.
} 
factors of the only primary boundary field in the Lee-Yang model computed in [2, 6] could also have been derived this way.

In principle, the agreement of the most singular term calculated from the form factor expansion with the quantum field expectation for some operator $\mathcal{O}$ leaves open the possibility of mixing with other fields of smaller weight (i.e. which are more relevant) in the sense that it allows that the form factor corresponds to $\mathcal{O}$ with an addition of an unspecified linear combination of operators with smaller weight, i.e. to an operator of the form

$$
\mathcal{O}+\sum_{l} C_{l} \mathcal{O}_{l}
$$

where the $\mathcal{O}_{l}$ are operators with scaling dimension less than that of $\mathcal{O}$. This problem was already noted in 3 . However, for the most relevant boundary field this issue is definitely not present which is reassuring for the determination of its form factors using the construction proposed above.

In the sinh-Gordon case the spectrum of conformal dimensions is unbounded from below, but the fact that the short-distance limit of the two-point function constructed from form factors is consistent with the leading part expected from the conformal operator product algebra and the exact vacuum expectation values eliminates some possibilities of such mixing. However, it still allows e.g. for

$$
\mathrm{e}^{k \frac{g}{2} \Phi(t, 0)}+\sum_{l>k} C_{l} \mathrm{e}^{l \frac{g}{2} \Phi(t, 0)}
$$

(where it was assumed that $k>0$ ).

Another restriction comes from the continuation to sine-Gordon theory. The parameter $k$ of the solution which gives the corresponding boundary exponential operator as

$$
\mathrm{e}^{k \frac{g}{2} \Phi(t, 0)}
$$

can take any real value, but the operators with $k \in \mathbb{Z}$ form a closed operator algebra, which is the minimal one consistent with the presence of the boundary potential in (2.3). Continuing analytically to the sine-Gordon theory with action (4.4), the $n$-particle form factors of these operators become the form factors of

$$
\mathrm{e}^{i k \beta \phi(t, 0)}
$$

with a many-particle state that consists exclusively of $n$ first breathers. The operators with $k= \pm 1$ are the most relevant ones in this family, and therefore they cannot mix with other operators at all; this property is expected to hold after continuing back to sinh-Gordon theory as well. In fact, these would be the operators for which there is the widest spectrum of subleading operators to choose from in (5.1), therefore the absence of mixing in their case is particularly striking.

The above arguments cannot be considered complete, but taken together they make it likely that there is eventually no operator mixing for the exponential fields in the boundary 
sinh-Gordon model at all. The basis provided by the solutions constructed in this paper is the only one for which the following property holds: for any member of the basis, the limiting form factor (in the sense of (3.11)) is not only a solution of the bulk form factor axioms, but also satisfies the asymptotic factorization condition (3.12). Note that this factorization property is needed for the derivation of the cumulant expansion (3.17) and it also ensures that the integrals in the expansion are convergent (cf. eqns. (3.15) and (3.16)) ). Certainly some deeper understanding of the boundary form factor bootstrap is needed in order to relate this condition to properties of relevant boundary primary fields, and to make the correspondence between form factor solutions and operators more precise.

\section{Acknowledgments}

I would like to thank F. Smirnov for a useful discussion, in particular for drawing my attention to the cumulant expansion; also Z. Bajnok and L. Palla for comments about the manuscript. This research was partially supported by the Hungarian research fund OTKA K60040 and also by a Bolyai János research scholarship.

\section{References}

[1] S. Ghoshal and A.B. Zamolodchikov, Int. J. Mod. Phys. A9 (1994) 3841-3886 (Erratum-ibid. A9 4353), hep-th/9306002.

[2] Z. Bajnok, L. Palla and G. Takács, Nucl. Phys. B750 (2006) 179-212, hep-th/0603171.

[3] M. Szôts and G. Takács, Nucl. Phys. B785 (2007) 211-233, hep-th/0703226.

[4] O.A. Castro-Alvaredo, J. Phys. A39 (2006) 11901-11914, hep-th/0606269.

[5] O.A. Castro-Alvaredo: Form factors of boundary fields for A(2)-affine Toda field theory, arXiv:0710.0501 [hep-th].

[6] M. Kormos and G. Takács: Boundary form factors in finite volume, arXiv: 0712.1886 [hep-th].

[7] A. Fring, G. Mussardo and P. Simonetti, Nucl.Phys. B393 (1993) 413-441, hep-th/9211053.

[8] E. Corrigan and A. Taormina, J.Phys. A33 (2000) 8739-8754, hep-th/0008237.

[9] S. Ghoshal, Int. J. Mod. Phys. A9 (1994) 4801-4810, hep-th/9310188.

[10] E. Corrigan: Boundary bound states in integrable quantum field theory, hep-th/0010094, in: Proceedings of 4th Annual European TMR Conference on Integrability Non-perturbative Effects and Symmetry in Quantum Field Theory, Paris, France, 7-13 Sep 2000. 
[11] Al.B. Zamolodchikov, talk at the 4th Bologna workshop on conformal and integrable models (unpublished).

[12] Z. Bajnok, G. Böhm and G. Takács, J. Phys. A35 (2002) 9333-9342, hep-th/0207079. Z. Bajnok, G. Böhm and G. Takács, Nucl. Phys. B682 (2004) 585-617, hep-th/0309119.

[13] F.A. Smirnov, Nucl. Phys. B337 (1990) 156-180.

[14] H. Babujian and M. Karowski, Int. J. Mod. Phys. A19S2 (2004) 34-49, hep-th/0301088.

[15] G. Delfino, P. Simonetti and J.L. Cardy, Phys. Lett. B387 (1996) 327-333, hep-th/9607046.

[16] A. Koubek and G. Mussardo, Phys. Lett. B311 (1993) 193-201, hep-th/9306044.

[17] S.L. Lukyanov and A.B. Zamolodchikov, Nucl. Phys. B493 (1997) 571-587, hep-th/9611238;

V.A. Fateev, S.L. Lukyanov, A.B. Zamolodchikov and Al.B. Zamolodchikov, Nucl. Phys. B516 (1998) 652-674, hep-th/9709034.

[18] V.A. Fateev, A.B. Zamolodchikov and Al.B. Zamolodchikov: Boundary Liouville field theory. 1. Boundary state and boundary two point function, hep-th/0001012.

[19] Al. B. Zamolodchikov, Int. J. Mod. Phys. A10 (1995) 1125-1150.

[20] Z. Bajnok, L. Palla and G. Takács, Nucl. Phys. B622 (2002) 565-592, hep-th/0108157. 